\title{
Simultaneous Determination of Seven Sesquiterpene Lactone Glucosides in Ixeris dentata by High-Performance Liquid Chromatography Coupled with Tandem Mass Spectrometry and their Antiviral Activities
}

\author{
SeonJu Park ${ }^{1}$, Nanyoung Kim ${ }^{1}$, Jun Hyung Park ${ }^{1}$, Sang-Won Lee ${ }^{2}$, Jae-Hyoung Song ${ }^{3}$, Hyun-Jeong Ko ${ }^{3}$, \\ Han-Jung Chae ${ }^{4}$, Hyung-Ryong Kim ${ }^{5}$ and Seung Hyun Kim ${ }^{1^{*}}$ \\ ${ }^{1}$ College of Pharmacy, Yonsei Institute of Pharmaceutical Science, Yonsei University, Incheon, Korea \\ ${ }^{2}$ Department of Herbal Crop Research, National Institute of Horticultural and Herbal Science, \\ Rural Development Administration, Chungbuk, Korea \\ ${ }^{3}$ Laboratory of Microbiology and Immunology, College of Pharmacy, Kangwon National University, Chuncheon, Korea \\ ${ }^{4}$ Department of Pharmacology and Institute of Cardiovascular Research, Sechool of Medicine, \\ Chonbuk National University, Chonbuk, Korea \\ ${ }^{5}$ Graduate School, DGIST, Daegu, Korea
}

Received: 27 March 2018; accepted: 15 May 2018

\begin{abstract}
Ixeris dentata (Thunb. ex Thunb.) Nakai (Asteraceae), a well-known edible vegetable in Asia, contains various bioactive secondary metabolites, including sesquiterpene lactones. In this study, a high-performance liquid chromatography-tandem mass spectrometry (HPLC-MS/MS) method has been developed and validated for simultaneous determination of seven sesquiterpene lactone glucosides isolated from the roots of $I$. dentata. In addition, these compounds were evaluated in terms of their antiviral activities against coxsackievirus B3 (CVB3) and human enterovirus 71 (EV71). The developed method was validated in terms of linearity $\left(R^{2}>0.9996\right)$, precision (RSD $<2.24 \%$ ), accuracy $(96.30-102.77 \%)$, and stability (RSD $<1.94 \%$ ) and successfully applied to the quantitation of the $I$. dentata root samples collected from six different regions of Korea. The content of sesquiterpene lactone glucosides varied significantly based on the region. For the antiviral activities, guaianolides with an ester group at C-8 (compounds 6 and 7) showed the most potent activities against CBV3, while germacranolide (compound 5) showed the most consistent antiviral activity against both CVB3 and EV71. The method was validated to be simple and reliable to simultaneously determine seven putative bioactive sesquiterpene lactone glucosides, the substantial chemotaxonomic markers, in I. dentata root samples.
\end{abstract}

Keywords: Ixeris dentata, guaianolides, germacranolide, HPLC-MS/MS, antiviral activity

\section{Introduction}

Ixeris dentata (Thunb. ex Thunb.) Nakai. (Asteraceae), called sowthistle, is a well-known edible wild vegetable in Asia. Its young leaves and roots are usually eaten cooked, while the very young leaves are eaten raw [1]. Reported chemical constituents of the genus Ixeris form seven major classes: sesquiterpene lactones, triterpenes and steroids, phenylpropanoids, phenols, amino acids, and fatty alcohol/acids [2]. In our previous studies, sesquiterpene glucosides isolated from $I$. dentata proved to exhibit significant pharmacological activities. For instance, ixerisoside A exhibited a meaningful inhibitory effect on lipopolysaccharideinduced nitric oxide production in BV-2 microglial cells [3]. In addition, 8-epiisolipidiol-3- $O$ - $\beta$-glucopyranoside and ixerin $\mathrm{F}$ were proven to be useful to prevent and to treat xerostomia [4]. In our continuing project to develop the roots of I. dentata as a dietary supplement, it is necessary to develop a simultaneous determination method of these bioactive compounds to assist the evaluation of quality control practice.

Several reports have been published about simultaneous determinations of sesquiterpene lactones in Cicerbita alpina via high-performance liquid chromatography with diode-array detection (HPLC-DAD) [5], in I. chinensis via HPLC with photo diode array (HPLC-PDA) [6], and in I. sonchifolia via HPLCquadrupole time-of-flight mass spectrometry (HPLC-QTOF-MS) [7]. Previous study also compared I. dentata, I. dentata var. albiflora, and $I$. sonchifolia to identify the main compounds

*Author for correspondence: kimsh11@yonsei.ac.kr present in extracts using HPLC [8]. In quality control applications, many studies have used high-performance liquid chromatography coupled to mass spectrometry (HPLC-MS) for qualitative and quantitative analyses; it is now widely accepted as the predominant tool for the qualification and quantification of natural products [9]. Among various MS spectrometers, triple quadrupole MS stands out as a fast, sensitive, and accurate quantification method for complex systems, such as natural product extracts $[10,11]$.

The goal of the present study was to develop and validate a method coupling HPLC with tandem mass spectrometry (HPLC$\mathrm{MS} / \mathrm{MS}$ ) for the simultaneous determination of seven sesquiterpene lactone glucosides, namely, 11 $\beta$,13-dihydrozaluzanin-3-O$\beta$-glucopyranoside (1), ixerin F (2), macrocliniside A (3), 8epiisolipidiol-3- $O$ - $\beta$-glucopyranoside (4), ixerin $\mathrm{H}(\mathbf{5})$, ixerisoside A (6), and ixerin M (7), in I. dentata extracts. To date, there have been no simultaneous analyses of sesquiterpene glucoses from I. dentata.

These sesquiterpene lactone glucosides were examined for their antiviral effects on coxsackievirus B3 (CVB3) and human enterovirus 71 (EV71) infection in vitro. CVB3, a member of the Picornaviridae family, is the most common human pathogen for viral myocarditis that leads to dilated cardiomyopathy, which may cause unexpected death in children and adolescents or endstage congestive heart failure in adults [12]. EV71, also a member of the Picornaviridae family, disrupts the central nervous system in humans and causes hand, foot, and mouth disease with significant morbidity and mortality [13]. Although many classes of natural products have been studied for their antiviral activities,

This is an open-access article distributed under the terms of the Creative Commons Attribution-NonCommercial 4.0 International License (https://creativecommons.org/licenses/by-nc/4.0/), which permits unrestricted use, distribution, and reproduction in any medium for non-commercial purposes, provided the original author and source are credited, a link to the CC License is provided, and changes - if any - are indicated. 
limited research has focused on sesquiterpene lactone $[14,15]$. In this study, a simple and efficient method was developed for simultaneous determination of seven sesquiterpene lactone glucosides from various regions in Korea, and these compounds were tested for their antiviral effects.

\section{Experimental}

2.1. Herbal Materials. I. dentata root samples were collected in Oct. 2015 and identified by Dr. Sang-Won Lee, a senior researcher in National Institute of Horticultural and Herbal Science, Rural Development Administration (RDA), Korea. Whole I. dentata plants were collected, and after removing the upper parts, only roots of the sample were washed, dried with air dryer at $50{ }^{\circ} \mathrm{C}$ for $6 \mathrm{~h}$, and cut. The samples were re-dried for $24 \mathrm{~h}$ and deposited at the Herbarium of Pharmacy, Yonsei Institute of Pharmaceutical Sciences, Yonsei University, Incheon, Korea (voucher specimen numbers: ID2014-01-ID2014-06).
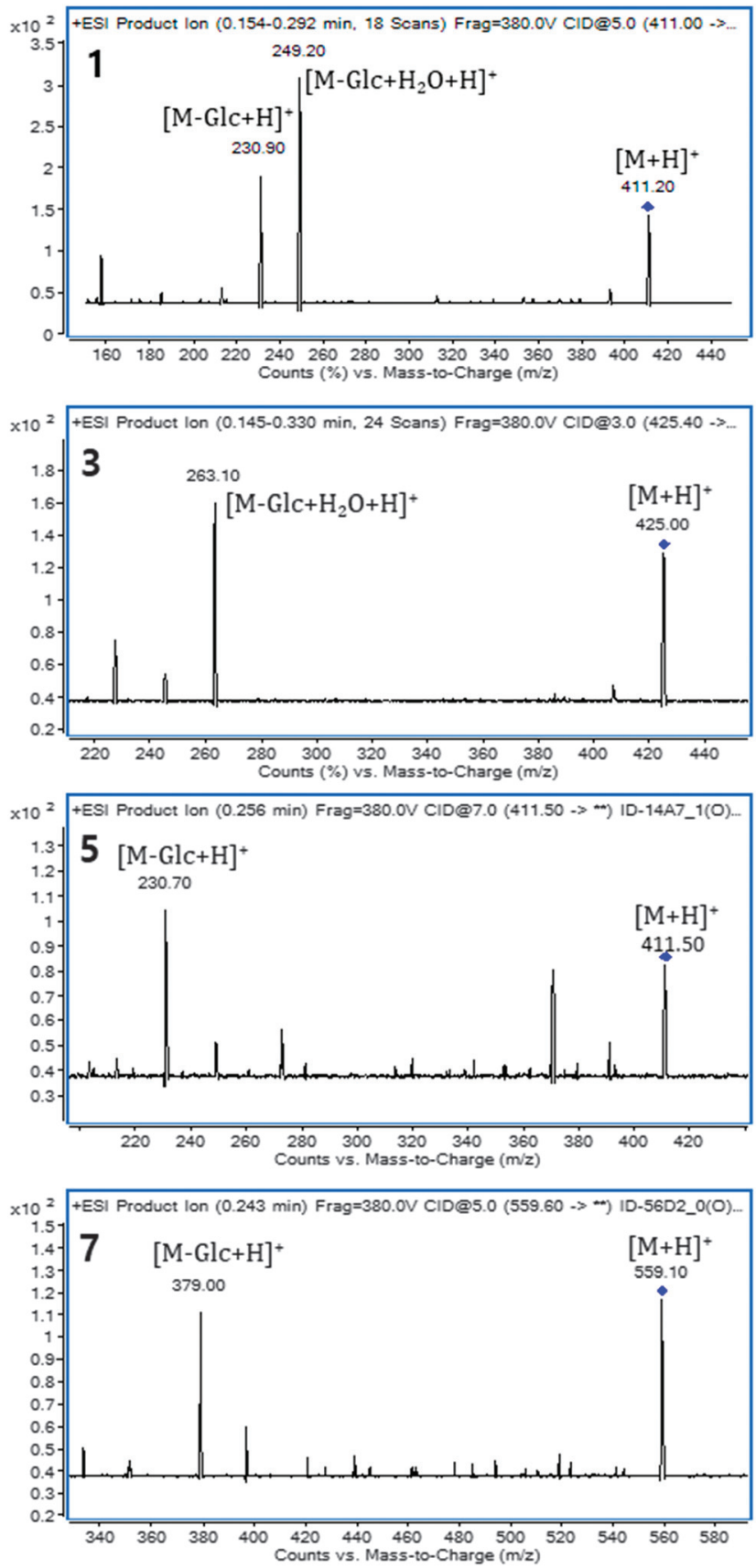

Figure 1. Representative MS/MS spectra of compounds 1-7
The reference standards of 11 $\beta, 13$-dihydrozaluzanin-3- $O$ $\beta$-glucopyranoside (1), ixerin F (2), macrocliniside A (3), 8epiisolipidiol-3- $O$ - $\beta$-glucopyranoside (4), ixerin $\mathrm{H}$ (5), ixerisoside A (6), and ixerin M (7) (Figure 1) were isolated and purified from I. dentata by various chromatographic techniques [3]. Comparison of the NMR and MS data with reported values in the literature led to the identification of these structures, and purities were determined to be $>98 \%$ by HPLC-DAD analysis based on a peak area normalization method.

2.2. Solvents and Reagents. The solvents, HPLC-grade acetonitrile and water, were purchased from J.T. Baker (Phillipsburg, NJ, USA), and HPLC-grade acetic acid from Sigma-Aldrich (St. Louis, MO, USA). Other reagents and chemicals were of analytical grade. A $0.2-\mu \mathrm{m}$ nylon membrane filter was purchased from Thermo Scientific (Schwerte, Germany). Antibiotic-antimycotic solution, trypsin-ethylenediaminetetraacetic acid (EDTA), fetal bovine serum (FBS), and minimum essential medium (MEM) were purchased from Gibco BRL (Invitrogen Life Technologies, Karlsruhe, Germany). Tissue culture plates
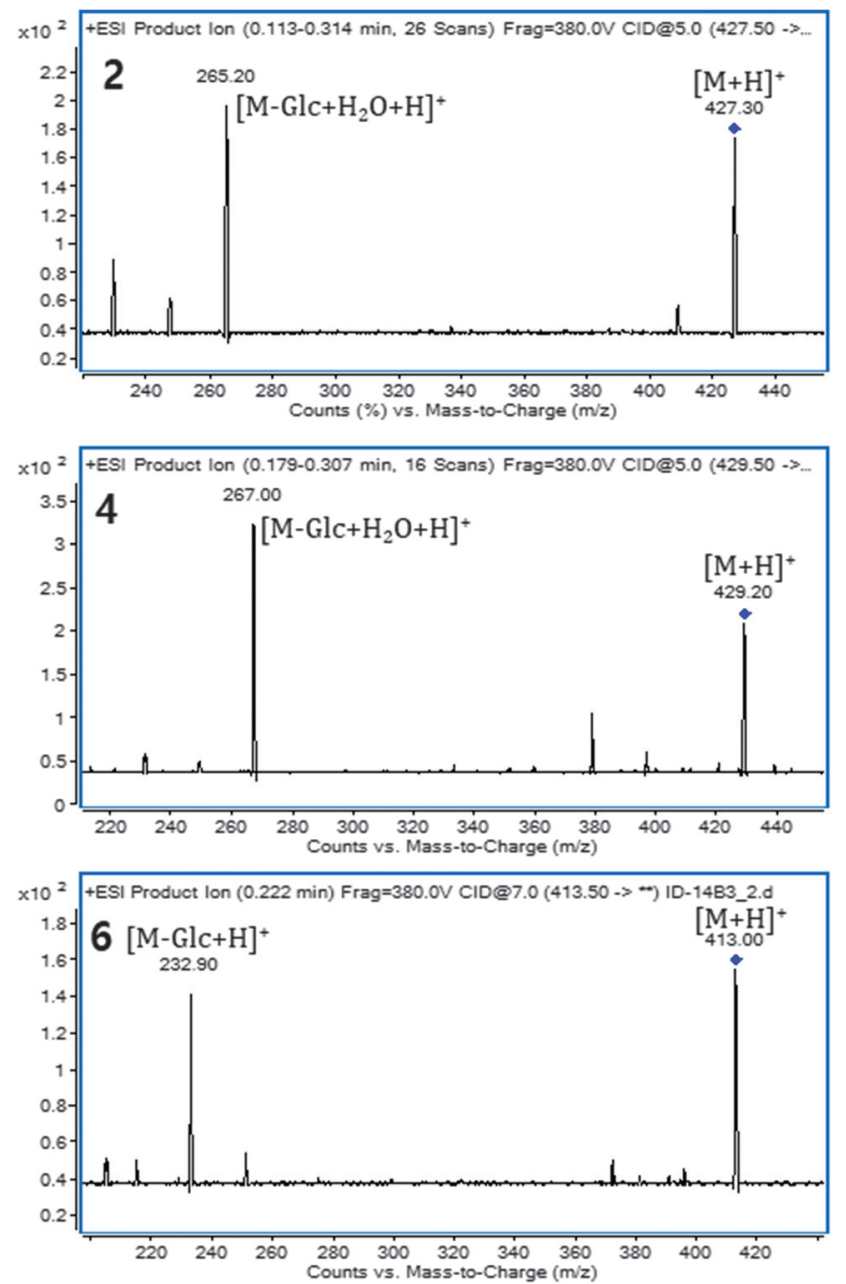
were purchased from Falcon (BD Biosciences, San Jose, CA, USA). Sulforhodamine B (SRB) was purchased from SigmaAldrich (St. Louis, MO, USA).

2.3. Sample Preparation. Pulverized dried roots (10 g) were extracted by an ultrasonicator with $50 \mathrm{~mL}$ of $100 \%$ methanol for $30 \mathrm{~min}$, filtered using Whatman filter paper No. 1 (Maidstone, UK), and then evaporated in vacuo. The extract was dissolved in $100 \%$ methanol at a concentration of $1 \mathrm{mg} / \mathrm{mL}$ and filtered through a $0.45-\mu \mathrm{m}$ PTFE syringe filter for analysis.

Individual seven compounds (1-7) were prepared at a concentration of $0.01 \mathrm{mg} / \mathrm{mL}$ in $100 \%$ methanol for standard solutions. Standard mixture containing $0.01 \mathrm{mg} / \mathrm{mL}$ of each compound was mixed and prepared by serial dilution to achieve appropriate concentrations with $100 \%$ methanol.

2.4. Instruments and Chromatographic Conditions. Chromatographic analysis was performed using an Agilent 1260 Series HPLC system (Agilent Technologies, Santa Clara, CA, USA) coupled to an Agilent 6410 triple quadrupole mass spectrometer equipped with an electrospray ionization (ESI) interface (Agilent Technologies, Santa Clara, CA, USA). Chromatographic separation was carried out at $30{ }^{\circ} \mathrm{C}$ on an YMC Hydrosphere C18 (4.6 mm $\times 150$ mm, $5 \mu \mathrm{m}$; YMC Co., Kyoto, Japan) column. The mobile phase consisted of $0.5 \%$ acetic acid in water (A) and acetonitrile (B) using a gradient elution of $7-11 \% \mathrm{~B}$ at $0-30 \mathrm{~min}$ and $11-40 \% \mathrm{~B}$ at $30-55 \mathrm{~min}$. The flow rate was kept at $0.5 \mathrm{~mL} / \mathrm{min}$. The injection volume was $10 \mu \mathrm{L}$. Quantification was performed using the positive ion multiple reaction monitoring (MRM) mode. The MS analyses were performed under the following operation parameters: both the auxiliary and sheath gases were nitrogen with a flow rate of $12 \mathrm{~L} / \mathrm{min}$. The drying gas temperature was set at $350{ }^{\circ} \mathrm{C}$, the fragmentor voltage was $380 \mathrm{~V}$, and the nebulizer pressure was set at 45 psi. Full-scan data acquisition and data-dependent acquisition scan event were performed from $\mathrm{m} / \mathrm{z} 200$ to 800 . The collision energy was adjusted from 3 to $7 \mathrm{~V}$ appropriate to the different analytes. The system was controlled by MassHunter software (Agilent Corporation, MA, USA).

2.5. Method Validation Parameter. The HPLC-MS/MS method was validated in terms of linearity, precision, accuracy, and stability according to the International Conference on Harmonization $(\mathrm{ICH})$ guidelines [16, 17]. Six-point calibration curves ranging from 0.2 to $5 \mu \mathrm{g} / \mathrm{mL}$ for each of the seven compounds were analyzed in triplicate. The limit of detection (LOD) and limit of quantification (LOQ) were determined based on the signal-to-noise ratio $(\mathrm{LOD}=3.3 \delta / S$ and LOQ $=10 \delta / S$, where $\delta$ is the standard deviation of the response and $S$ is the slope of the calibration curve). The precision was validated by the determination of intra- and inter-day variances. The intra-day variability was assayed at six concentrations on the same day, and the inter-day variability was assayed at three concentrations for three consecutive days. The precision of the developed method was expressed by the relative standard deviation (RSD). The accuracy was determined by the method of standard addition. The diluted sample solution of the methanolic extract of I. dentata $(1 \mathrm{mg} / \mathrm{mL})$ was spiked with the mixture of standard compounds in the ratios of $2: 1,1: 1$, and $1: 2$. Then, the resultant samples were analyzed by the proposed method, and triplicate experiments were carried out. Accuracy was represented as average recoveries (\%) of the spiked analyte concentrations. For stability assay, a crude extract that was obtained directly by the RDA was analyzed immediately after preparation and then reassayed after storage at the room temperature for $2,4,8,12$, and $24 \mathrm{~h}$.

2.6. Antiviral Activity. The CVB 3 and EV71 were obtained from the division of vaccine research of the Korea Centre Disease Control and Prevention and were propagated at $37^{\circ} \mathrm{C}$ in Vero cells (ATCC, Manassas, VA, USA). Vero cells were maintained in MEM supplemented with 10\% FBS and $0.01 \%$ antibiotic-antimycotic solution. The cells were incubated in MEM supplemented with $10 \%$ FBS and $0.01 \%$ antibioticantimycotic solution in a humidified atmosphere of $5 \% \mathrm{CO}_{2}$ at $37{ }^{\circ} \mathrm{C}$. Cells were detached using trypsin-EDTA. Five subsequent cycles of subculturing were done before the cells were incubated with the seven isolated standard compounds [18]. Antiviral activity was assayed by employing the SRB method using cytopathic effect (CPE) reduction as recently reported [19]. To evaluate the activity, $2 \times 10^{4}$ cells per well of Vero cells were seeded using MEM supplemented with 10\% FBS and 0.01\% antibiotic-antimycotic solution onto a 96-well culture plate and incubated for $24 \mathrm{~h}$. Then, the medium was removed, the cells were washed, and the diluted virus suspension $(0.09 \mathrm{~mL})$, containing a $50 \%$ cell culture infective dose $\left(\mathrm{CCID}_{50}\right)$ of the virus, was added to cells to produce a $50 \%$ CPE within $48 \mathrm{~h}$ after infection. Next, MEM containing $10 \mu \mathrm{M}$ of the compounds $(0.1 \mathrm{~mL})$ was added and incubated at $37{ }^{\circ} \mathrm{C}$ in $5 \% \mathrm{CO}_{2}$ for 2 days until the $50 \% \mathrm{CPE}$ was achieved. Subsequently, the 96-well plates were washed once with $200 \mathrm{~mL}$ of PBS. Icecold $70 \%$ acetone in water $(100 \mu \mathrm{L})$ was added to each well and incubated for $30 \mathrm{~min}$ at $-20{ }^{\circ} \mathrm{C}$. After removing the $70 \%$ acetone, the plates were dried in a drying oven at $55{ }^{\circ} \mathrm{C}$ for $30 \mathrm{~min} .0 .4 \%(\mathrm{w} / \mathrm{v}) \mathrm{SRB}$ in $1 \%$ acetic acid solution $(100 \mu \mathrm{L})$ was added to each well and left for $30 \mathrm{~min}$ at room temperature. The SRB solution was then removed, and the plates were washed 5 times with $1 \%$ acetic acid in water before oven-drying at $55{ }^{\circ} \mathrm{C}$. Bound SRB was then solubilized with $10 \mathrm{mM}$ unbuffered Tris-base (Sigma) solution $(100 \mu \mathrm{L})$. After $30 \mathrm{~min}$, the absorbance was read at $540 \mathrm{~nm}$ using a VERSAmax microplate reader (Molecular Devices, Palo Alto, CA, USA) with a reference absorbance at $620 \mathrm{~nm}$. The percent protection achieved by the test compound in the HRV1B-, CVB3-, and PR8-infected cells was calculated using the following equation: antiviral activity index $=$ $\left[\mathrm{OD}_{\mathrm{t}}-\mathrm{OD}_{\mathrm{c}}\right] /\left[\left(\mathrm{OD}_{\mathrm{t}}\right)_{\text {mock }}-\mathrm{OD}_{\mathrm{c}}\right] \times 100 \%$.

The absorbances obtained from Vero cells infected with 50\% tissue culture infective dose $\left(\mathrm{TCID}_{50}\right)$ viruses were used as controls. $\mathrm{OD}_{\mathrm{t}}$ is the optical density measured with a given test compound in virus-infected cells, $\mathrm{OD}_{\mathrm{c}}$ is the optical density measured for the control untreated virus-infected cells, and $\left(\mathrm{OD}_{\mathrm{c}}\right)_{\text {mock }}$ is the optical density measured for control untreated non-infected cells.

\section{Results and Discussion}

3.1. Antiviral Activities. To search for new and effective antiviral compounds, the biological activity of all isolated compounds was evaluated against CVB3 and EV71 infection. CBV3 and EV71, members of the Picornaviridae family that are currently divided into nine genera, are causative agents of human diseases. However, there are no safe and effective vaccines or therapeutics to treat viruses classified within the Picornaviridae family [20]. In this regard, many studies have been conducted to find antiviral components from many traditional medicinal plants. Among these potential plants, we have focused on the I. dentata extract that already demonstrated antiviral activity against surrogate viruses in vitro for hepatitis B and hepatitis C [21].

The antiviral activity was investigated by an SRB method using CPE reduction at the final concentration of $10 \mu \mathrm{M}$ of each compound. Sesquiterpene lactones, with flavonoids and lignans, are regarded as a major class of natural products with a variety of biological activities. However, limited antiviral research has focused on sesquiterpene lactone, especially with sesquiterpene lactone glucoses [14, 15]. Table 1 summarizes data on the cell viability of the tested sesquiterpene lactone glucosides against CVB3 and EV71.

In CVB3, compounds 6 and 7, the class of guaianolides with an ester group at C-8, showed a stronger antiviral activity 
Table 1. Antiviral activity index of sesquiterpene lactone glucosides isolated from I. dentata against CVB3 and EV71

\begin{tabular}{lcc}
\hline Compound & \multicolumn{2}{c}{ Antiviral activity index (\%) } \\
\cline { 2 - 3 } & \multicolumn{1}{c}{ CVB3 } & EV71 \\
\hline $\mathbf{1}$ & $2.15 \pm 1.11$ & $38.00 \pm 0.50$ \\
$\mathbf{2}$ & $1.85 \pm 1.10$ & $83.51 \pm 1.73$ \\
$\mathbf{3}$ & $5.45 \pm 1.19$ & $28.02 \pm 0.32$ \\
$\mathbf{4}$ & $15.81 \pm 3.60$ & $14.77 \pm 1.05$ \\
$\mathbf{5}$ & $33.73 \pm 1.93$ & $61.70 \pm 1.12$ \\
$\mathbf{6}$ & $41.87 \pm 2.14$ & $-8.89 \pm 0.98$ \\
$\mathbf{7}$ & $45.53 \pm 2.24$ & $-3.03 \pm 0.82$ \\
\hline
\end{tabular}

with a significant antiviral activity index of 41.87 and $45.53 \%$ compared to compounds $\mathbf{1}-\mathbf{3}$ (1.85-5.45\%), guaianolides without ester group nor hydroxyl group at C-8. In the contrast, compounds $\mathbf{2}$ and $\mathbf{5}$ showed more potent antiviral activities than the other samples against EV71 (83.51 and 61.70\%, respectively, Table 1). Unfortunately, we could not identify the common structural features between these two compounds. Compound 5, a class of germacranolide, showed a clinical potential against CVB3 and EV71, since it exhibited the most consistent antiviral activities against the two viruses tested (33.73 and $61.70 \%$ of antiviral activity index for CBV3 and EB71, respectively). These results indicate that sesquiterpene lactone glucoses possess protective effects and provide potential candidates for the development of novel drugs to treat CVB3 and EV71 infections. Previous study reported that sesquiterpenes inhibit viral DNA synthesis by membrane-mediated mechanisms [22]. In addition, in discussing their biological activity, it is generally accepted that the $\alpha$-methylene- $\gamma$-lactone moiety (Michael acceptor) of sesquiterpene lactones is the most important structural features. However, previous research showed that these two structural requirements are not essential for potent antiviral activity [15]. Therefore, a detailed mechanism study with sesquiterpene lactone glucoses is required to clarify their antiviral activities.

3.2. Development of HPLC-MS/MS Analysis. Different mobile phases (methanol-water, acetonitrile-water, methanolacid aqueous solution, and acetonitrile-acid aqueous solution) were examined and compared to develop the chromatographic analysis. An eluting solvent system of acetonitrile- $0.5 \%$ aqueous acetic acid was chosen to provide acceptable separation within a run time of $55 \mathrm{~min}$.

In a preliminary study, the methanolic extract of $I$. dentata was analyzed in Q1 scan mode. Based on the resulting chromatogram, seven major sesquiterpene lactone glucosides (1-7) were chosen as marker compounds of $I$. dentata extracts. Due to higher transcript level which ultimately leads to the biosynthesis of sesquiterpenes, the roots of $I$. dentata accumulate various sesquiterpenes [23]. Therefore, the roots of I. dentata are majorly composed of sesquiterpene derivatives. In addition, sesquiterpene lactones are a particular feature of Asteraceae, and one of the advantages of using them as chemotaxonomic markers is their lower volatility [24].

The MS/MS profiles of 1-7 were acquired to determine precursor ions and product ions for use in the MRM mode (Figure 2). The target compounds were favorably ionized in the positive ion mode to yield the protonated molecular ion $[\mathrm{M}+\mathrm{H}]^{+}$. To obtain the richest relative abundance of precursor ions and product ions, the collision energy for each compound was separately determined (Table 2). The MRM transitions were then optimized and chosen to be $m / z 411.5[\mathrm{M}+\mathrm{H}]^{+} \rightarrow 230.7[\mathrm{M}-\mathrm{Glu}+\mathrm{H}]^{+}$for 1, $m / z 427.5[\mathrm{M}+\mathrm{H}]^{+} \rightarrow 265.2\left[\mathrm{M}-\mathrm{Glu}+\mathrm{H}_{2} \mathrm{O}+\mathrm{H}\right]^{+}$for 2,
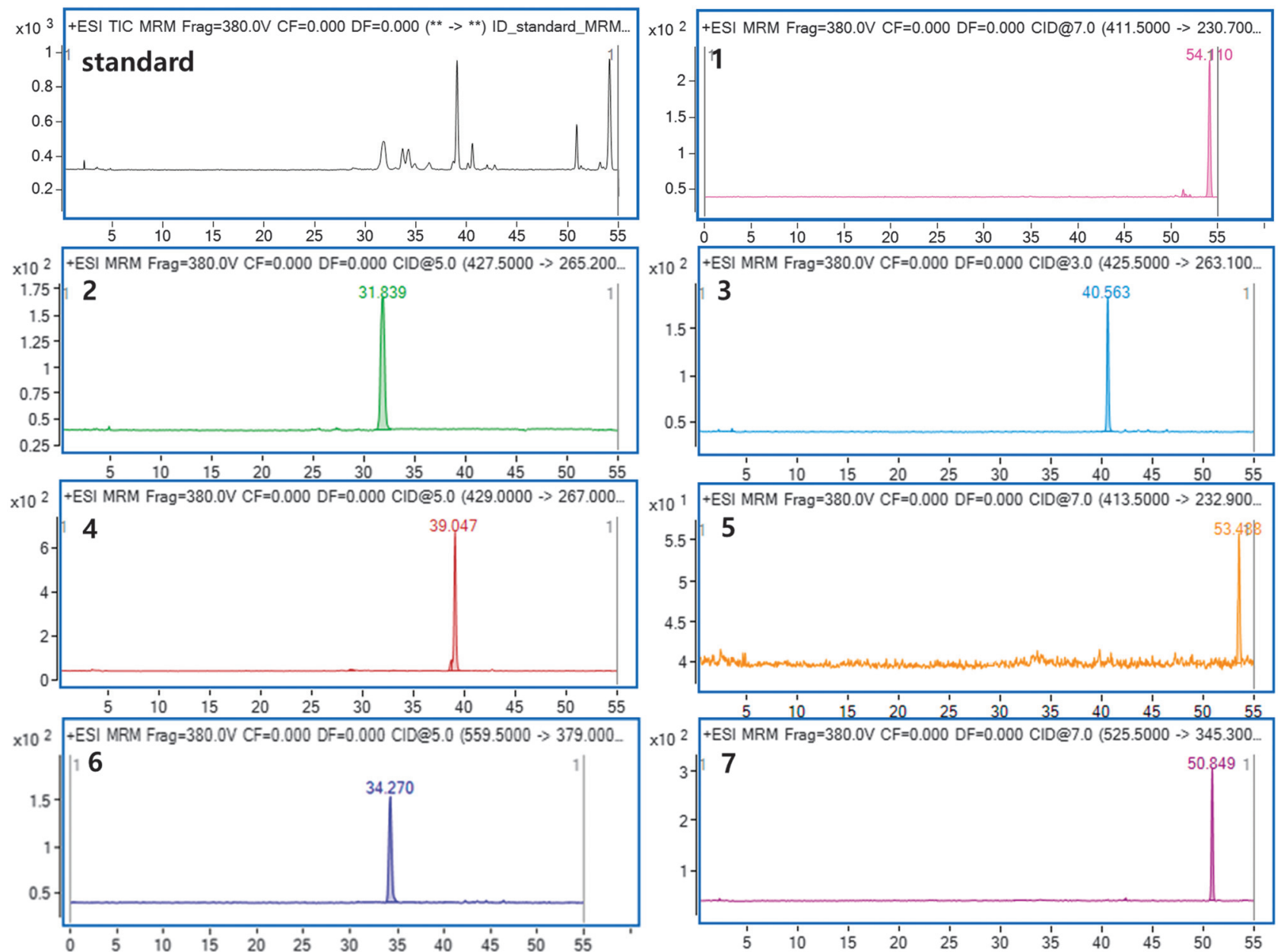

Figure 2. Representative MS/MS chromatograms of compounds 1-7 
Table 2. Analytical data for the sesquiterpene lactone glucosides (1-7)

\begin{tabular}{|c|c|c|c|c|c|c|c|c|}
\hline Compd. & RT (min) & {$[\mathrm{M}+\mathrm{H}]^{+}(m / z)$} & Quantitative ion $(\mathrm{m} / \mathrm{z})$ & $\mathrm{CE}(\mathrm{V})$ & Calibration curve equation & $R^{2}$ & LOD $(\mu \mathrm{g} / \mathrm{mL})$ & LOQ $(\mu \mathrm{g} / \mathrm{mL})$ \\
\hline 1 & 54.110 & 411.5 & 230.9 & 5 & $y=613999 x+149$ & 0.9999 & 0.18 & 0.53 \\
\hline 2 & 31.839 & 427.5 & 265.2 & 5 & $y=505819 x+216$ & 0.9999 & 0.12 & 0.35 \\
\hline 3 & 40.563 & 425.5 & 263.1 & 3 & $y=210294 x+30$ & 0.9996 & 0.07 & 0.21 \\
\hline 5 & 53.488 & 413.5 & 232.9 & 7 & $y=118047 x+33$ & 0.9997 & 0.06 & 0.20 \\
\hline 6 & 34.270 & 559.5 & 379.0 & 5 & $y=70283 x+3$ & 0.9998 & 0.10 & 0.31 \\
\hline 7 & 50.849 & 525.5 & 345.3 & 7 & $y=121806 x+24$ & 0.9998 & 0.27 & 0.82 \\
\hline
\end{tabular}

RT - retention time; CE - collision energy; LOD - limits of detection; LOQ - limits of quantification.

$m / z 425.5[\mathrm{M}+\mathrm{H}]^{+} \rightarrow 263.1\left[\mathrm{M}-\mathrm{Glu}+\mathrm{H}_{2} \mathrm{O}+\mathrm{H}\right]^{+}$for $\mathbf{3}, \mathrm{m} / z$ $429.0[\mathrm{M}+\mathrm{H}]^{+} \rightarrow 267.0\left[\mathrm{M}-\mathrm{Glu}+\mathrm{H}_{2} \mathrm{O}+\mathrm{H}\right]^{+}$for $4, \mathrm{~m} / z$ $413.5[\mathrm{M}+\mathrm{H}]^{+} \rightarrow 232.9[\mathrm{M}-\mathrm{Glu}+\mathrm{H}]^{+}$for $\mathbf{5}, \mathrm{m} / 2559.5[\mathrm{M}+$ $\mathrm{H}]^{+} \rightarrow 379.0[\mathrm{M}-\mathrm{Glu}+\mathrm{H}]^{+}$for $\mathbf{6}$, and $m / z 525.5[\mathrm{M}+\mathrm{H}]^{+} \rightarrow$ $345.3[\mathrm{M}-\mathrm{Glu}+\mathrm{H}]^{+}$for 7. Retention time and MS information are shown for each analyte, including $[\mathrm{M}+\mathrm{H}]^{+}$, quantitative ions, and CE in Table 2.

3.3. Method Validation of Developed Method. The developed method was validated regarding linearity, precision, accuracy, and stability according to ICH Q2 guidelines [16, 17]. Acceptable linear correlation and high sensitivity under these conditions were confirmed by the correlation coefficients $\left(r^{2}\right.$, $0.9996-0.9999)$, the LOD ranging from 0.06 to $0.27 \mu \mathrm{g} / \mathrm{mL}$, and the LOQ ranging from 0.20 to $0.82 \mu \mathrm{g} / \mathrm{mL}$ (Table 2). When compared with the representative chromatograms of the seven

Table 3. Analytical method precision of the sesquiterpene lactone glucosides $(\mathbf{1}-7)(n=6)$

\begin{tabular}{|c|c|c|c|c|}
\hline \multirow[t]{2}{*}{ Compound } & \multicolumn{2}{|c|}{ Intra-day } & \multicolumn{2}{|c|}{ Inter-day } \\
\hline & $\begin{array}{c}\text { Concentration } \\
(\mu \mathrm{g} / \mathrm{mL})\end{array}$ & $\begin{array}{c}\text { Precision } \\
(\% \text { RSD })\end{array}$ & $\begin{array}{c}\text { Concentration } \\
(\mu \mathrm{g} / \mathrm{mL})\end{array}$ & $\begin{array}{c}\text { Precision } \\
(\% \text { RSD })\end{array}$ \\
\hline \multirow[t]{6}{*}{1} & 5 & 0.11 & 3 & 0.50 \\
\hline & 3 & 0.12 & & \\
\hline & 2 & 0.57 & 2 & 0.42 \\
\hline & 1 & 0.67 & & \\
\hline & 0.5 & 0.21 & 1 & 0.72 \\
\hline & 0.2 & 1.24 & & \\
\hline \multirow[t]{6}{*}{2} & 5 & 0.05 & 3 & 0.37 \\
\hline & 3 & 0.10 & & \\
\hline & 2 & 0.16 & 2 & 0.39 \\
\hline & 1 & 0.28 & & \\
\hline & 0.5 & 0.14 & 1 & 0.47 \\
\hline & 0.2 & 0.11 & & \\
\hline \multirow[t]{6}{*}{3} & 5 & 0.05 & 3 & 0.42 \\
\hline & 3 & 0.12 & & \\
\hline & 2 & 0.13 & 2 & 0.23 \\
\hline & 1 & 0.32 & & \\
\hline & 0.5 & 1.15 & 1 & 0.65 \\
\hline & 0.2 & 1.67 & & \\
\hline \multirow[t]{6}{*}{4} & 5 & 0.01 & 3 & 0.21 \\
\hline & 3 & 0.15 & & \\
\hline & 2 & 0.25 & 2 & 0.20 \\
\hline & 1 & 0.95 & & \\
\hline & 0.5 & 0.04 & 1 & 0.94 \\
\hline & 0.2 & 2.24 & & \\
\hline \multirow[t]{6}{*}{5} & 5 & 0.14 & 3 & 0.16 \\
\hline & 3 & 0.15 & & \\
\hline & 2 & 0.08 & 2 & 0.69 \\
\hline & 1 & 0.23 & & \\
\hline & 0.5 & 0.84 & 1 & 0.11 \\
\hline & 0.2 & 0.32 & & \\
\hline \multirow[t]{6}{*}{6} & 5 & 0.16 & 3 & 0.34 \\
\hline & 3 & 0.46 & & \\
\hline & 2 & 0.17 & 2 & 0.19 \\
\hline & 1 & 0.67 & & \\
\hline & 0.5 & 0.25 & 1 & 0.39 \\
\hline & 0.2 & 1.60 & & \\
\hline \multirow[t]{6}{*}{7} & 5 & 0.03 & 3 & 0.16 \\
\hline & 3 & 0.15 & & \\
\hline & 2 & 0.12 & 2 & 0.36 \\
\hline & 1 & 0.55 & & \\
\hline & 0.5 & 0.46 & 1 & 0.44 \\
\hline & 0.2 & 0.65 & & \\
\hline
\end{tabular}

compounds in the standard mixture and methanolic extracts of I. dentata, no significant interference with analyte detection was observed in any of the methanolic extracts tested (supplementary data), suggesting high specificity of the developed method. The intra- or inter-day precisions calculated as RSD were within the range of $0.01-2.24 \%$ or $0.11-0.94 \%$, respectively, and these data are shown in Table 3. The accuracy of the analysis for sesquiterpene lactone glucosides 1-7 varied from $96.30 \%$ to $102.77 \%$ (Table 4). These results indicate that the present method is acceptable for accurate and precise determination of analytes. The stability of the seven analytes in the sample solution was evaluated for $24 \mathrm{~h}$ under ambient conditions. The content of each compound in the sample at different time points showed no significant differences, and the RSD values were $<1.94 \%$ (Table 5), which indicated that the compounds and the sample were relatively stable for at least $24 \mathrm{~h}$ after preparation.

3.4. Quantitative Analysis of Samples. The developed analytical method was subsequently used to analyse the presence of 1-7 in I. dentata root samples collected from six different regions of Korea in the same season. The proposed HPLC-MS/MS method separated the sesquiterpene lactone glucosides 1-7 in different $I$. dentata samples within a run time of $55 \mathrm{~min}$ (Figure 3 in the supplementary data). Representative extracted ion chromatograms of each I. dentata extract are shown in the supplementary data. All compounds except for $\mathbf{5}$ belong to the class of guaianolides, and the guaianolides were detected in six samples as shown in Table 6. However, the data indicated that guaianolide content varied significantly based on region. For instance, compound $\mathbf{6}$ content, which is the dominant component in the samples, varied from 9.18 to $30.26 \mu \mathrm{g} / \mathrm{g}$, which shows more than three-fold variation. Similar variations were also observed in other guaianolides. However, compound 5, the germacranolide, was detected only in two regions.

Table 4. Analytical method accuracy for the sesquiterpene lactone glucosides $(\mathbf{1}-\mathbf{7})(\mu \mathrm{g} / \mathrm{mL}, n=3)$

\begin{tabular}{lcccr}
\hline Compound & \multicolumn{2}{c}{ Concentration of analytes } & \multicolumn{1}{c}{$\begin{array}{c}\text { Accuracy } \\
\text { (\% recovery) }\end{array}$} \\
\cline { 2 - 4 } & Original & Spiked & Found & \\
\hline $\mathbf{1}$ & 0.061 & 1.333 & 1.408 & $100.97 \pm 0.40$ \\
$\mathbf{2}$ & 0.445 & 1.333 & 1.777 & $99.65 \pm 0.28$ \\
$\mathbf{3}$ & 0.340 & 1.333 & 1.710 & $102.77 \pm 1.35$ \\
$\mathbf{4}$ & 0.067 & 1.333 & 1.361 & $97.04 \pm 1.39$ \\
$\mathbf{5}$ & 0.119 & 1.333 & 1.403 & $96.30 \pm 1.81$ \\
$\mathbf{6}$ & 0.157 & 1.333 & 1.507 & $101.22 \pm 1.45$ \\
$\mathbf{7}$ & 0.122 & 1.333 & 1.450 & $99.59 \pm 2.50$ \\
\hline
\end{tabular}

Table 5. Stability of seven sesquiterpene lactone glucosides (1-7) in the I. dentata sample at room temperature $(\mu \mathrm{g} / \mathrm{g}, n=3)$

\begin{tabular}{|c|c|c|c|c|c|c|c|c|}
\hline \multirow[t]{2}{*}{ Compound } & \multicolumn{6}{|c|}{ Content } & \multirow{2}{*}{$\begin{array}{r}\text { Average } \\
\text { content }\end{array}$} & \multirow{2}{*}{$\begin{array}{l}\text { RSD } \\
(\%)\end{array}$} \\
\hline & $0 \mathrm{~h}$ & $2 \mathrm{~h}$ & $4 \mathrm{~h}$ & $8 \mathrm{~h}$ & $12 \mathrm{~h}$ & $\overline{24 h}$ & & \\
\hline 1 & 5.18 & 5.02 & 5.18 & 5.20 & 5.06 & 5.10 & 5.12 & 1.34 \\
\hline 2 & 2.76 & 2.68 & 2.78 & 2.76 & 2.70 & 2.72 & 2.78 & 1.51 \\
\hline 3 & 2.02 & 2.08 & 2.00 & 2.06 & 2.02 & 2.04 & 2.04 & 1.45 \\
\hline 4 & 8.30 & 8.30 & 8.06 & 8.48 & 8.38 & 8.22 & 8.28 & 1.73 \\
\hline 5 & 1.38 & 1.36 & 1.34 & 1.38 & 1.40 & 1.36 & 1.38 & 1.53 \\
\hline 6 & 10.5 & 10.76 & 10.76 & 10.90 & 10.90 & 10.64 & 10.74 & 1.46 \\
\hline 7 & 6.40 & 6.74 & 6.64 & 6.60 & 6.68 & 6.48 & 6.6 & 1.94 \\
\hline
\end{tabular}


Table 6. Contents $(\mu \mathrm{g} / \mathrm{g}, n=3)^{a}$ of seven sesquiterpene lactone glucosides (1-7) in the $I$. dentata samples collected from different regions of Korea

\begin{tabular}{|c|c|c|c|c|c|c|}
\hline Compound & $\begin{array}{l}\text { Chungbuk Eumseong } \\
\text { (S1) }\end{array}$ & $\begin{array}{c}\text { Chungbuk Goesan } \\
\text { (S2) }\end{array}$ & $\begin{array}{c}\text { Chungnam Dangjin } \\
\text { (S3) }\end{array}$ & $\begin{array}{c}\text { Gangwon Chuncheon } \\
\text { (S4) }\end{array}$ & $\begin{array}{c}\text { Gangwon Jinbu } \\
\text { (S5) }\end{array}$ & $\begin{array}{c}\text { Gyeonggi Yangpyeong } \\
\text { (S6) }\end{array}$ \\
\hline 1 & $2.66 \pm 0.10$ & $2.88 \pm 0.12$ & $2.54 \pm 0.22$ & $1.62 \pm 0.10$ & $2.78 \pm 0.16$ & $1.96 \pm 0.04$ \\
\hline 2 & $2.88 \pm 0.32$ & $1.00 \pm 0.06$ & $4.18 \pm 0.58$ & $15.9 \pm 0.24$ & $10.74 \pm 0.58$ & $4.40 \pm 0.58$ \\
\hline 3 & $1.90 \pm 0.11$ & $3.16 \pm 0.30$ & $2.84 \pm 0.25$ & $6.14 \pm 0.04$ & $4.15 \pm 0.09$ & $17.91 \pm 0.50$ \\
\hline 4 & $4.21 \pm 0.08$ & $24.44 \pm 0.19$ & $8.91 \pm 0.50$ & $16.82 \pm 0.15$ & $12.41 \pm 1.61$ & $9.91 \pm 0.40$ \\
\hline 5 & $2.41 \pm 0.14$ & n.d. & n.d. & n.d. & n.d. & $13.8 \pm 0.08$ \\
\hline 6 & $14.28 \pm 0.29$ & $30.26 \pm 0.24$ & $9.18 \pm 0.23$ & $31.74 \pm 0.14$ & $22.34 \pm 0.11$ & $25.90 \pm 0.15$ \\
\hline 7 & $7.26 \pm 0.14$ & $5.02 \pm 0.12$ & $5.88 \pm 0.20$ & $6.04 \pm 0.12$ & $12.06 \pm 0.17$ & $11.50 \pm 0.25$ \\
\hline
\end{tabular}

Moreover, 11 $\beta$,13-dihydrozaluzanin-3-O- $\beta$-glucopyranoside (1), which showed moderate antifungal activity, exhibited the most similar content in six different regions among sesquiterpene glucosides $(1.62-2.88 \mu \mathrm{g} / \mathrm{g})$. Potential therapeutic compounds for preventing and treating xerostomia, ixerin $\mathrm{F}(2)$, and 8 epiisolipidiol-3- $O$ - $\beta$-glucopyranoside (4) were the highest in I. dentata from Gangwon Chuncheon $(15.9 \mu \mathrm{g} / \mathrm{g})$ and Chungbuk Goesan $(12.22 \mu \mathrm{g} / \mathrm{g})$, respectively. The roots from Gangwon Chuncheon contained a relatively high amount of both compounds 2 and $\mathbf{4}$. Consequently, it can be a potential therapeutic plant source for the treatment of xerostomia. The roots from Gyeonggi Yangpyeong showed relatively high macrocliniside A (3) and ixerin M (7) contents, which showed strong cytotoxicity. These results showed that there is substantial regional variability in sesquiterpene lactone glucosides in I. dentata across Korea and suggested that environmental conditions including geographical location notably contributed to substantial variations of I. dentata root samples. The HPLC-MS/MS method provided reliable quantitative analysis of the multi-component samples and proved to be readily applicable as a quality control for extract for the analysis of bioactive compounds from I. dentata.

\section{Conclusion}

Sesquiterpene lactones are a particular feature of Asteraceae, and the roots of $I$. dentata are known to accumulate various sesquiterpenes. In this study, a simple and sensitive HPLC-MS/MS method was established in order to simultaneously determine sesquiterpene substantial chemotaxonomic markers of the roots of $I$. dentata. A newly developed method was validated in terms of linearity, precision, accuracy, and stability and successfully applied to six regional root samples in Korea. These results showed that there is substantial regional variability in sesquiterpene lactone glucosides in I. dentata across Korea and provided the feasibility of our newly developed method for the quality control of the $I$. dentata root samples. In addition, these substantial chemotaxonomic compounds were tested for their antiviral activities against CVB3 and EV71 infection in vitro. Antiviral results indicated that sesquiterpene lactone glucoses possess protective effects and provide potential candidates for the development of novel drugs to treat CVB3 and EV71 infections.

Acknowledgement. This work was carried out with the support of Cooperative Research Program for Agriculture Science \& Technology Development (Project No. PJ01158104) funded by the Rural Development Administration, Republic of Korea.

\section{References}

1. Ha, S. C.; Won, S. W.; Sook, J. L. Phytochemistry 1994, 35, 1583.

2. Zhang, Y. C.; He, C. N.; Chew, E. H. Chem. Biodivers. 2013, 10, 1373

3. Park, S.; Nhiem, N. X.; Lee, T. H.; Kim, N.; Kim, S. Y.; Chae, H.-J.; Kim, S. H. Bioorg. Med. Chem. Lett. 2015, 25, 4562.

4. Lee, H. Y.; Lee, G. H.; Kim, H. K.; Kim, S. H.; Park, K. P.; Chae, H. J.; Kim, H. R. Food Chem. Toxicol. 2013, 62, 739.

5. Fusani, P.; Zidorn, C. J. Food Compos. Anal. 2010, 23, 658.

6. Wang, Q.; Ao, W.; Ying, X.; Meng, G.; Wu, X.; Tai, W. J. Food Compos. Anal. 2012, 20, 872 .

7. Shi, P.; Zhang, Y.; Qu, H.; Fan, X. Phytochem. Anal. 2011, 22, 66.

8. Karki, S.; Park, H.-J.; Nugroho, A.; Kim, E. J.; Jung, H. A.; Choi, J. S. J. Med. Food 2015, 18, 83 .

9. Constant, H. L.; Beecher, C. W. Nat. Prod. Lett. 1995, 6, 193.

10. Churchwell, M. I.; Twaddle, N. C.; Meeker, L. R.; Doerge, D. R. J. Chromatogr. B 2005, 825, 134

11. Han, Y. M.; Jang, M.; Kim, I. S.; Kim, S. H.; Yoo, H. H. J. Sep. Sci. 2015, 38, 2260

12. Martino, T. A.; Liu, P.; Sole, M. J. Circ. Res. 1994, 74, 182.

13. Lin, T. Y.; Chu, C.; Chiu, C. H. J. Infect. Dis. 2002, 186, 1161.

14. Hwang, D. R.; Wu, Y. S.; Chang, C. W.; Lien, T. W.; Chen, W. C.;

Tan, U. K.; Hsu, J. T. A.; Hsieh, H. P. Bioorg. Med. Chem. Lett. 2006, 14, 83.

15. Ozcelik, B.; Gurbuz, I.; Karaoglu, T.; Yesilada, E. Microbiol. Res. 2009, 164, 545 .

16. ICH (1995) Q2A: Text on validation of analytical procedures: terms and definitions. In: International Conference on Harmonization, Fed. Reg. (60 FR 11260)

17. ICH (1997) Q2B: Validation of analytical procedures: methodology. In: International Conference on Harmonization, Fed. Reg. (62 FR 27463)

18. Song, J. H.; Choi, H. J.; Song, H. H.; Hong, E. H.; Lee, B. R.; Oh, S. R.; Choi, K.; Yeo, S. G.; Lee, Y. P.; Cho, S. J. Ginseng Res. 2014, 38, 173.

19. Choi, H. J.; Kim, J. H.; Lee, C. H.; Ahn, Y. J.; Song, J. H.; Baek, S. H.; Kwon, D. H. Antivir. Res. 2009, 81, 77.

20. Cheng, A.; Fung, C. P.; Liu, C. C.; Lin, Y. T.; Tsai, H. Y.; Chang, S. C.; Chou, A. H.; Chang, J. Y.; Jiang, R. H.; Hsieh, Y. C. Vaccine 2013, 31, 2471.

21. Jacob, J. R.; Korba, B. E.; You, J. E.; Tennant, B. C.; Kim, Y. H. J. Altern. Complement. Med. 2004, 10, 1019.

22. Jassim, S.; Naji, M. A. J. Appl. Microbiol. 2003, 95, 412

23. Kim, M. Y.; Chang, Y. J.; Bang, M. H.; Baek, N. I.; Jin, J.; Lee, C. H.; Kim, S. U. J. Plant Biol. 2005, 48, 178.

24. Barbero, F.; Maffei, M. Biodiversity and chemotaxonomic significance of specialized metabolites CRC Press: Boca Raton, FL, USA, 2016. 